\title{
Computer-Aided Recognition of ABC Transporters Substrates and Its Application to the Development of New Drugs for Refractory Epilepsy
}

\author{
Manuel Couyoupetrou ${ }^{1,2}$, Melisa E. Gantner ${ }^{1}$, Mauricio E. Di Ianni ${ }^{1}$, Pablo H. Palestro ${ }^{1}$, \\ Andrea V. Enrique ${ }^{1}$, Luciana Gavernet ${ }^{1}$, María E. Ruiz ${ }^{1}$, Guido Pesce ${ }^{2}$, Luis E. Bruno-Blanch ${ }^{1}$, \\ and Alan Talevi ${ }^{2}$
}

${ }^{1}$ Medicinal Chemistry/Laboratory of Bioactive Research and Development (LIDeB), Department of
Biological Sciences, Faculty of Exact Sciences, University of La Plata, La Plata, Argentina;
${ }^{2}$ Department of Pharmacology, National Institute of Medications (INAME), Administración Nacional
de Medicamentos, Alimentos y Tecnología Médica (ANMAT), Buenos Aires, Argentina

Abstract: Despite the introduction of more than 15 third generation antiepileptic drugs to the market from 1990 to the moment, about one third of the epileptic patients still suffer from refractory to intractable epilepsy. Several hypotheses seek to explain the failure of drug treatments to control epilepsy symptoms in such patients. The most studied one proposes that drug resistance might be related with regional overactivity of efflux transporters from the ATP-Binding Cassette (ABC) superfamily at the

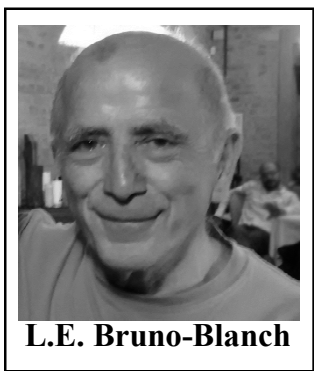
blood-brain barrier and/or the epileptic foci in the brain. Different strategies have been conceived to address the transporter hypothesis, among them inhibiting or down-regulating the efflux transporters or bypassing them through a diversity of artifices. Here, we review scientific evidence supporting the transporter hypothesis along with its limitations, as well as computer-assisted early recognition of $\mathrm{ABC}$ transporter substrates as an interesting strategy to develop novel antiepileptic drugs capable of treating refractory epilepsy linked to $\mathrm{ABC}$ transporters overactivity.

Keywords: ABC transporters, ABCB1, ABCG2, Antiepileptic drugs, Breast Cancer Resistance Protein, Drug discovery, P-glycoprotein, Refractory epilepsy, Transporter hypothesis.

\section{INTRODUCTION}

\subsection{Drug Resistant Epilepsy: Definition and Current Explanations}

Epilepsy is the most frequent chronic brain disorder, affecting about 50 million people worldwide [1]. While drug therapy is the treatment of choice and can successfully treat (i.e. provide sustained seizure freedom) about $70 \%$ of people with epilepsy [1], the remaining $30 \%$ of the patients suffer from drug resistant, refractory or intractable epilepsy [2], i. e. the failure to achieve seizure freedom through adequate trials of two tolerated appropriately chosen antiepileptic drug (AED) schedules [3] (note that a clear, universal definition of refractory epilepsy will be fundamental to approach the limitations of the neurobiological explanations to refractory epilepsy later in this article). This scenario has not changed substantially in spite of the introduction of more than 15 third generation AEDs from 1990 to the moment [4], a fact that has led to a decrease in the industrial interest in the development of new compounds for epilepsy [5].

*Address correspondence to this author at the Department of Biological Sciences, Faculty of Exact Sciences, University of La Plata, La Plata, Argentina; Tel: +542214235333 ext 41; E-mail: lbb@biol.unlp.edu.ar
Biological mechanisms underlying drug resistant epilepsy have not been fully elucidated yet [5], though there exist to the day five hypotheses that try to explain the nature of this phenomenon: the transporter hypothesis [6, 7], the target hypothesis [7, 8], the neural network hypothesis [9], the gene variant hypothesis [10] and the intrinsic severity hypothesis [11]. Historically speaking, the transporter and target hypotheses have been conceived earlier and have therefore been more extensively explored from an experimental viewpoint.

The transporter hypothesis sustains that drug resistant epilepsy may be a consequence of the local overactivity of ATP-Binding Cassette (ABC) transporters at the blood-brain barrier (BBB) and/or the epileptic foci. A more detailed overview on the evidence and limitations of this hypothesis is provided in the next subsection. The target hypothesis proposes that the reduced sensitivity to AEDs might be linked to acquired modifications in the structure and/or functionality of AED targets. While some years back constitutive alterations of drug transporters or targets were also considered within the scope of the transporter and target hypothesis, respectively $[8,12]$, leading experts in the field now seem to prefer to categorize intrinsic alterations (genetic variants) of drug targets within the gene variant hypothesis [5]. The gene variant hypothesis, however, also covers other 
possible genetic causes of drug resistance (e.g. polymorphic variants of drug biotransformation enzymes). In the frame of this novel classification scheme, only acquired modifications in drug transporters or targets would be contained by the transporter or target hypothesis. Though at first sight this distinction may appear as a trivial classification problem, the nature of the pharmacokinetic or pharmacodynamic alteration could have a profound impact on the clinical approach to the drug resistance issue. While a genetic cause of pharmacoresistance might currently be detected through simple diagnostic tests even before starting the treatment, acquired modifications linked to the pathophysiology of the disease are more difficult to prove and still require more invasive procedures (e.g. surgery resection). Recently, a possible role of epigenetics in drug resistance epilepsy has also been suggested (establishing a sixth hypothesis for refractory epilepsy that might serve to expand the biological basis to other previous hypotheses such as the transporter and the target hypotheses), though to the moment the experimental basis supporting this mechanism remains scarce [13].

The neural network hypothesis maintains that recurrent episodes of excessive neural activity lead to plastic alterations and remodeling of the neural network; abnormal networking might in turn relate to the drug resistance phenomena. The hypothesis is supported by the fact that surgical resection of the seizure focus frequently results in seizure freedom [5]. As a matter of fact, epilepsies of structural cause are linked to drug resistance and abnormal brain imaging, and a good surgical outcome (seizure freedom) is associated to complete surgical resection of the epileptic focus or lesion, pre-surgical presence of lesions in MRI scans and the network complexity [14]. The differences between the alterations in brain plasticity in responsive and non-responsive patients are yet to be elucidated [9].

At last, the intrinsic severity hypothesis relies on epidemiologic studies showing that the single most important factor linked to the prognosis of epilepsy is the number of seizures at the epilepsy onset. Again, the biological basis of disease severity are however no fully understood to the moment [15], so currently the influence of the intrinsic severity hypothesis on treatment choice or treatment development is limited.

It has been pointed out that none of the previous hypotheses provides a full or universal explanation to nonresponsive patients with epilepsy: a given hypothesis might be applicable to a particular subgroup of patients or, alternatively, some patients could require multiple hypotheses to explain their non-responsiveness [5, 12, 16]. The network hypothesis seems so far as the more holistic explanation to drug resistance, since some of the other explanations (e.g. the target hypothesis) could be applied in its context [5]. It is worth underlining that the treatment approach should be highly dependent on the drug resistance mechanisms present in a particular patient.

\subsection{Strengths and Weaknesses of the Transporter Hypothesis of Drug Resistant Epilepsy}

In eukaryotes, $\mathrm{ABC}$ transporters are transmembrane efflux transporters (they export their substrates from the cell) and they are characterized by broad-substrate specificity/ polispecificity $[17,18]$. They are preferentially expressed in barrier tissues (gut, BBB) and elimination organs (liver, kidneys), limiting the absorption and biodistribution and favoring the elimination of their substrates. Besides their role in the traffic of physiologic compounds (e.g. cholesterol or amyloid beta) they also take part in multi-drug resistance phenomena or pathogenesis in a diversity of disorders [1820]. Though most of the research on ABC transporters has focused on the first historically identified member of the superfamily (P-glycoprotein, Pgp or P-gp or ABCB1 or MDR1), attention of the scientific and medical community has more recently been given to other members, prominently MRPs (ABCCs) and Breast Cancer Resistance Protein (BCRP, ABCG2).

Preclinical validation of the transporter hypothesis for drug resistant epilepsy has been achieved, since drug resistance in animal models of refractory epilepsy has been reverted by co-administration of $\mathrm{ABC}$ transporters inhibitors. The first steps towards a proof of concept of the potential role of $\mathrm{ABC}$ transporters was delivered by Potschka et al. back in 2001 [21]. Using in vivo microdialysis in rats, the authors showed that the levels of carbamazepine in the extracellular fluid of the cerebral cortex could be enhanced through local perfusion of Pgp inhibitor verapamil and MRP1/2/5 inhibitor probenecid. Some time later, the same researchers proved that co-administration of probenecid (50 $\mathrm{mg} / \mathrm{kg})$ and phenytoin $(6.25 \mathrm{mg} / \mathrm{kg})$ resulted in a clear increase of phenytoin anticonvulsant effect in electrically kindled rats (a 90\% increase in the threshold for generalized seizures) [22]. Neither $50 \mathrm{mg} / \mathrm{kg}$ probenecid nor $6.25 \mathrm{mg} / \mathrm{kg}$ phenytoin exerted significant anticonvulsant effect when given alone. It was discussed that such raise in the seizure threshold was unlikely to result from additive effects of the chosen subanticonvulsant doses. Interestingly, the inhibition of MRP2 (which is located in the apical membrane of endothelial cells and thus opposes to drug penetration in the brain) results in a significant increase of drug levels in the brain which was not secondary to alterations in peripheral drug pharmacokinetics. Similar results were later obtained in the focal pilocarpine model of limbic seizures [23]: while ip administration of oxcarbazepine $100 \mathrm{mg} / \mathrm{kg}$ to rats did not prevent seizures, co-administration of verapamil or probenecid resulted in complete protection. The severity of pilocarpine-induced seizures severity was not affected by perfusion of any of the inhibitors alone. Though highly valuable, this initial works had two important limitations: a) the use of first-generation, weak and unspecific modulators of $\mathrm{ABC}$ transporters and; b) experiments were performed on animal groups with no discrimination between responder and non-responder subgroups. The first issue was later solved through the use of third-generation, specific inhibitor of Pgp tariquidar. Van Vliet et al. used a chronic epileptic rat model and showed that, while phenytoin alone did not achieve complete suppression of spontaneous seizures, coadministration of phenytoin and tariquidar led to an almost complete seizure control [24]. Inhibition of Pgp by tariquidar increased the phenytoin brain-to-plasma ratio; it was also shown that the maximal administered doses of tariquidar did not exert anticonvulsant activity per se. The effect of coadministration of tariquidar on seizure control reverted after 
four days, suggesting that tolerance to tariquidar was developed. Definitive preclinical proof of concept was obtained by co-administration of tariquidar to epileptic drug resistant animals associated to up-regulated Pgp expression [25]. The key innovation in this study was the introduction of a protocol to discriminate responsive and nonresponsive animals to phenobarbital (Fig. 1). When co-administering tariquidar, five out of six non-responders became seizurefree or displayed a reduction in seizure of at least $50 \%$. Similar results were obtained in the 3-mercaptopropionic acid model of refractory epilepsy, which is associated to Pgp upregulation at the $\mathrm{BBB}$, astrocytes and neurons [26]. While 3-mercaptopropionic acid epileptic rats showed significantly lower hippocampal phenytoin concentrations compared to the control group, pre-treatment of such animals with the Pgp inhibitor nimodipine led to enhanced hippocampal phenytoin bioavailability (the drug hippocampal bioavailability was, in fact, even higher than in control animals, suggesting other possible interactions between phenytoin and nimodipine). It should be noted, however, that verapamil add-on therapy failed to enhance seizure control in a study on 11 phenobarbital resistant dogs; in fact, some animals showed a tendency to worsening of seizure control [27]. These results highlight the potential importance of inter-species differences and the necessity to validate the transporter hypothesis using appropriate clinical trials. Even so, clinical proof-of-concept remains elusive, as will be discussed next.

Regarding clinical data, plenty of evidence has accumulated over the years showing high expression levels of $\mathrm{ABC}$ transporters at the neurovascular unit of nonresponder patients [28-35]. It should be commented, however, that most of these studies compared brain samples from patients with intractable epilepsy that had been subjected to surgical removal of the epileptic focus with specimens of human brain with no history of seizures. While brain tissue from epileptic drug-responsive patients would be a more suitable control, such control samples are usually unavailable since the invasive procedure to attain them is ethically unacceptable in responsive epileptic patients. This limitation has fortunately been overcome in more recent studies using positron emission tomography (PET) scans [36, 37] which showed that the plasma-to-brain transport rate constant $\mathrm{K}_{1}$ for $\left[{ }^{11} \mathrm{C}\right]$ verapamil and $(\mathrm{R})-\left[{ }^{11} \mathrm{C}\right]$ verapamil tends to be lower in different brain regions of drug resistant epileptic patients compared with seizure-free patients and healthy individuals. Whole brain $\mathrm{K}_{1}$ was increased in both healthy subjects and pharmacoresistant patients after tariquidar administration. The results from the study using (R)- $\left[{ }^{11} \mathrm{C}\right]$ verapamil [36] are particularly relevant since some of the limitations emerging from the diversity of factors affecting radiotracer kinetics [38, 39] are addressed. These are extremely important steps toward validation of the transporter hypothesis, though definite proof would require reversal of drug resistance (improvement in seizure control) after blocking ABC transporters. Anecdotal cases of refractory patients who have shown improvement when AEDs were co-administered with verapamil have been reported [40-43], but it is not clear yet if the observed results could be a consequence of the intrinsic anticonvulsant activity of verapamil (e.g. through modulation of calcium influx in neurons) or another effect of this drug on the AED pharmacokinetics. More recently, a pilot study was conducted on seven children with drug resistant epilepsy [44]. The patients received verapamil as add-on therapy to baseline AED. Three individuals with genetically determined Dravet syndrome showed a partial response to adjunctive verapamil; another patient with Dravet syndrome but no known mutation showed partial seizure control during 13 months followed by seizure worsening. Two subjects with structural epilepsy and one with Lennox-Gastaut displayed no improvement. Though the number of patients that took part in the study is very limited, the results are in line with the idea that some therapeutic interventions might be more effective in certain subgroups of non-responders. Later, a double-blind, randomized, single-centered trial (initial sample size $=22$ ) showed mild benefits of verapamil in comparison to placebo as add-on therapy for refractory

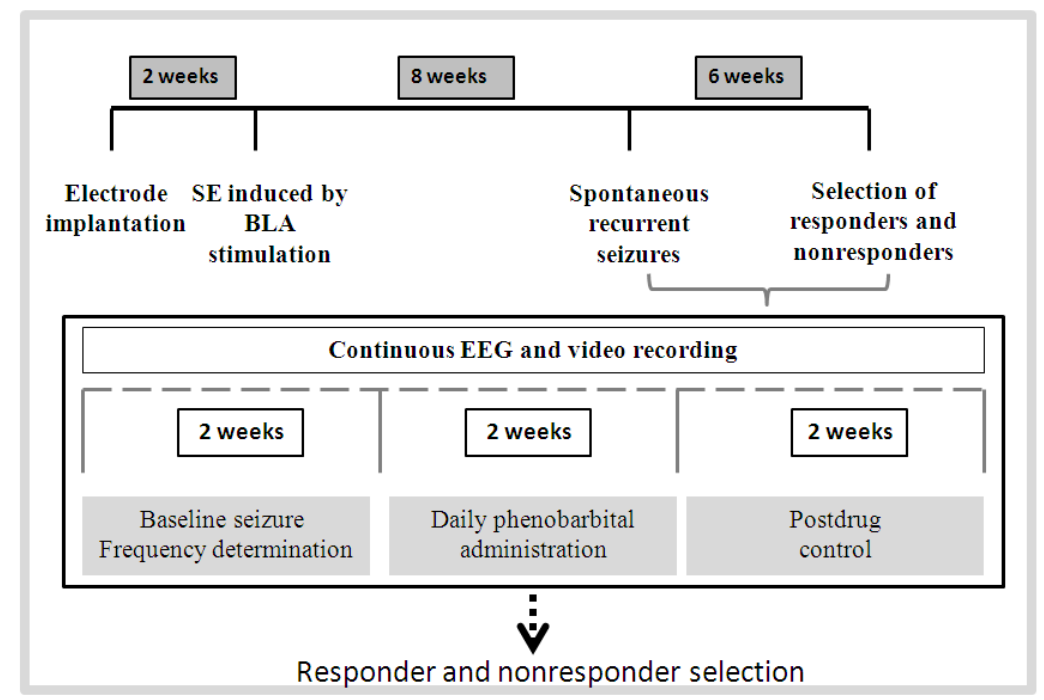

Fig. (1). Schematic illustration of selection of drug refractory rats from BLA model by prolonged administration of phenobarbital. SE= status epilepticus. BLA= prolonged electrical stimulation of the basolateral amygdala. 
epilepsy for a subset of the participants [45]. Randomized multi-centered control trials and studies addressing the effect of selective inhibitors of P-gp with no intrinsic activity are still necessary to gain definitive clinical evidence for the transporter hypothesis. Regarding the association between genetic variants of $\mathrm{ABC}$ transporters and drug resistant epilepsy, studies outcomes are controversial or sometimes inconclusive; while former meta-analysis failed to establish and association between $\mathrm{ABCB} 1$ variants and refractory epilepsy [46], subgroup analysis in more recent ones have suggested associations in Asian and Caucasian subjects [4749], thus contributing to the validity of the hypothesis.

The main argument against the transporter hypothesis seems to be that not all AEDs are in fact Pgp substrates. Although apparently conflictive evidence exists regarding which AEDs are substrates and which are not [50, 51], results are highly dependent on the experimental setting, including type of assay (in vivo, ex vivo or in vitro, human versus animal models, concentration equilibrium transport assay or non-equilibrium conditions). Still, it seems safe to say that at least some AEDs are unlike Pgp substrates. Some considerations should be taken into account to reach a conclusion regarding the assigned category (substrate or non-substrate). Possible inter-species variability in substrate specificity should not be excluded. In relation to in vitro permeability assays, bi-directional transport assays in presence and absence of a selective Pgp inhibitor might lack sensitivity since directional transport might be masked by the contribution of passive diffusion. The magnitude of this effect depends on the substrate assayed concentration/s, the transporter expression levels in the cell culture, the affinity between the drug and the transporter and the physicochemical features of the test drug (e.g. permeability), among other factors [52]. Starting the assay with identical concentrations of drug on both sides of the cell monolayer (concentration equilibrium transport assay, CETA) removes the concentration gradient, eliminating net diffusion and enhancing the assay sensitivity $[53,54]$.

Even if it has been demonstrated in appropriate models that some available AEDs are not Pgp substrates, does this undermine the transporter hypothesis as explanation drug resistance in epilepsy? Not necessarily. First, Pgp is one among many other efflux transporters possibly involved in refractory epilepsy. While most of the studies to determine the directional transport of AEDs have focused on Pgp, some of the antiepileptic agents are recognized and translocated by other members of the ABC superfamiliy. For instance, the role of $\mathrm{ABCG} 2$ in the drug resistance phenomena to AEDs might have been overlooked: while previous work seemed to suggest that several AEDs were not recognized by ABCG2 [49], more recent studies using double knock-out Mdrla/1b(-/-)/Bcrp $(-/-)$ mice and the CETA model suggest otherwise $[55,56]$. It is also relevant to note that proteomics studies have shown ABCG2 as the transporter with highest basal expression levels at the BBB of healthy subjects [57, 58], which underlines the convenience of assessing recognition by other ABC transporters apart from Pgp when designing novel AEDs. Furthermore, due to the partial overlapping of the substrate specificity of different members of the superfamiliy (which together with reported co-expression and co-localization patterns points to a cooperative role in the disposition of common substrates) [59-61], the role of a certain ABC transporter might be concealed due to the function of others, requiring complex models to study the phenomena. The difficulties to quantify the levels of expression of a given transporter in different regions of the brain of an epileptic patient who has not been subjected to a surgical intervention/resection, and the uncertainties regarding the ability of experimental models to reflect the absolute and relative expression levels of the different $\mathrm{ABC}$ efflux transporters at the epileptic foci and the BBB (expression levels which might well be highly patientdependent and highly dynamic) contribute to the difficulty of assessing unequivocally the influence of a given transporter in the regional AED bioavailability in the brain.

Apart from the need to contemplate the separate and concerted contributions of different ABC transporters to the efflux of AEDs from CNS, the current definition of refractory epilepsy itself suggest that the transporter hypothesis may hold even if known AEDs are recognized by ABC transporters. Since the definition indicates that a patient should be considered unresponsive after failure of two well tolerated and appropriately chosen and used AED trials, the key to the preceding reasoning lies in what is considered an appropriate drug choice. The definition of drug resistant epilepsy weakens the transporter hypothesis if and only if one of the two appropriate therapeutic interventions was in fact a non-substrate for $\mathrm{ABC}$ transporters. Presently, in absence of definitive clinical proof of the transporter hypothesis, it is not standard protocol to try at least one AED not recognized by $\mathrm{ABC}$ transporters; thus, to the moment the quality of substrate or non-substrate is not related to the appropriateness. If the transporter hypothesis were validated in at least a subgroup of the unresponsive patients, then a method for patient selection capable of identifying patients that may benefit from therapeutic strategies targeting efflux transport will be necessary; furthermore, patient selection should also be considered when designing clinical trials to study the clinical relevance of the transporter-associated resistance [62], excluding other sources of drug resistance as possible confounders.

\subsection{Possible Therapeutic Solutions to Transported- mediated Refractory Epilepsy}

There are a number of possible therapeutic solutions that could be and are being explored in consequence with the transporter hypothesis.

Inhibition of $\mathrm{ABC}$ transporters by adding on transporter inhibitors has been already proposed as a possible therapeutic solution to efflux-mediated drug resistant cancer. However, clinical trials to support this approach have so far been disappointing [16, 18, 62 and refs therein] owing to severe safety issues. The reader should bear in mind the physiologic role of $\mathrm{ABC}$ transporters as a general detoxification mechanism and their involvement in the traffic of endogenous substrates, which discourages the use of add-on inhibitors in the context of long-term drug treatments (such as the used in epilepsy). The potential effects of such inhibitors in the pharmacokinetics of other drugs should also be considered in 
a polymedication scenario, due to the high probability of adverse drug interactions. The connection between $\mathrm{ABC}$ transporter dysfunction and neurodegenerative diseases such as Parkison's and Alzheimer's diseases can be quoted as an example of the potential risk posed by chronic inhibition of this efflux systems [63-65]. Moderate or week inhibitors of $\mathrm{ABC}$ transporters thus emerge as possible solutions. So do therapeutic agents directed to the signaling cascade that regulates efflux transporters expression [62]. Such option might prove useful to prevent or ameliorate drug- or diseaseinduced up-regulation of transporters function, e.g. through activation of nuclear receptors or through pro-inflammatory signals, respectively. An extensive review on such approaches can be found in the excellent articles from Potschka [62, 66].

In the second place one may mention the use of a Trojan horse stratagem to deliver therapeutic levels of the $A B C$ transporters substrates to the epileptic focus, avoiding the recognition of the efflux pumps. Particulate delivery systems (mainly, pharmaceutical nanocarriers) can be included in this category [67, 68]. Interestingly, this approach allows encapsulating efflux pump substrate AEDs of clinical use within advanced delivery systems. Thus, the transference of such technologies to the clinical practice is expected to be more straightforward than other alternatives described in this section. Provided that safe delivery vectors are used, since the safety and efficacy of the pharmaceutical active ingredient have already been demonstrated, this strategy implies better chances of surviving clinical trials. In line with the preceding approach we can mention the design of prodrugs of AEDs either lacking affinity for ABC transporters or displaying affinity for influx transporters that could compensate the efflux pumps influence on BBB permeability. Though a diversity of prodrugs of approved AEDs have been conceived, the interaction of most of them with efflux pumps has not been assessed yet $[66,68]$.

The design of novel AEDs which are not recognized by ABC transporters and the early screening during drug development to discard substrates (thus considering efflux pumps as anti-targets) constitute interesting but underexplored alternative solutions. A scheme illustrating the different strategies overviewed in this subsection is presented in (Fig. 2). The following section will overview recent studies focused on this last approximation.

Screening during CNS drug development guarantees that high affinity substrates of BBB efflux transporters are not selected as lead compounds.

\section{IN SILICO SCREENING TO FIND THERAPEUTIC SOLUTIONS TO DRUG RESISTANT EPILEPSY}

The Medicinal Chemistry group from the National University of La Plata has implemented a cascade protocol integrating in silico (ligand- and structure-based), in vitro and in vivo models to detect potential new treatments for efflux transporter-associated refractory epilepsy. The protocol starts with high-throughput cost-efficient in silico screening tools and progressively advances to more expensive models with lower throughput, ending in preclinical models of drug-resistant epilepsy that will not be discussed in detail here. A schematic flux diagram of the protocol is displayed in (Fig. 3). Each step is covered separately under the correspondent subheading, below these lines.

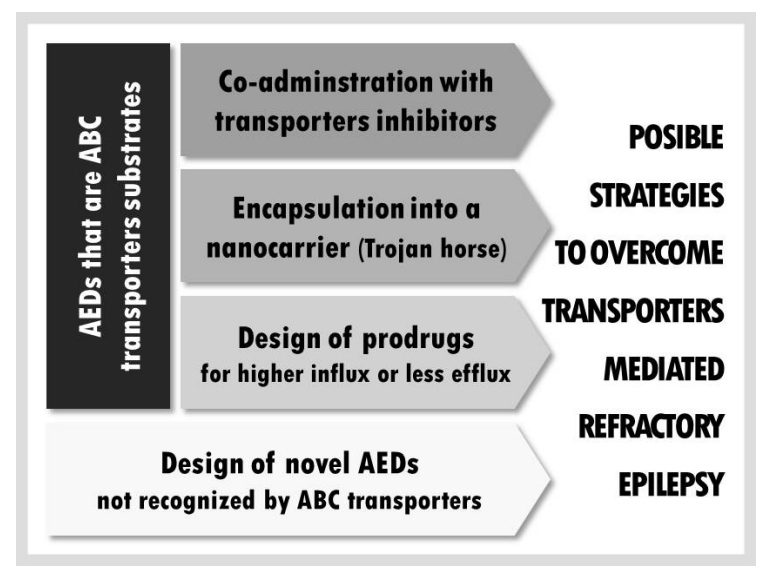

Fig. (2). Summary of the therapeutic strategies overviewed in section 1.3.

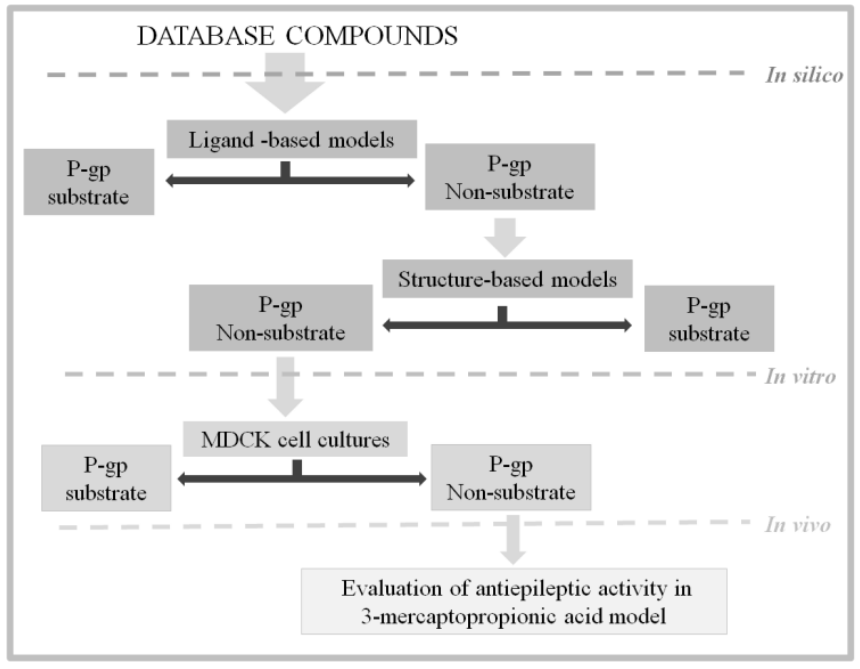

Fig. (3). Cascade protocol to screen for potential therapeutics for drug resistance epilepsy related to P-gp upregulation.

\subsection{In silico Models to Identify ABC Transporters Substrates. Applications to AEDs Screening}

A large number of computational models to detect potential substrates of $\mathrm{ABC}$ transporters have been reported, from pharmacophores to machine learning algorithms [69-71 and refs therein]. Many of those models have been derived from structural homologous series, thus being capable of making accurate predictions in their local chemical space but lacking general applicability. Others lack experimental validation of the predictive ability. While initially attention was drawn to Pgp and, particularly, the prediction of inhibitory activity, most recently, as the relevance of other efflux pumps is recognized and the inhibitors entering clinical trials fail, the focus has been gradually shifting 
towards other members of the $\mathrm{ABC}$ superfamily and the prediction of transport [71, 72]. Most of these models show accuracy around $80 \%$, which reflects the challenge posed by the multiplicity of binding mechanisms, the related polyspecificity and the high experimental variability of available data; such demanding proposition for modeling has led some authors to propose ensemble learning as a potential solution $[70,73,74]$. The reports of applications of such models to the selection of novel AEDs as potential treatment for drug resistant epilepsy are, to date, scarce.

In this regard, back in 2011 Di Ianni and colleagues reported a 3-model ensemble of 2D QSAR classifiers capable of differentiating P-gp substrates from nonsubstrates [75]. For this purpose, a 250-compound dataset including $104 \mathrm{P}$-gp substrates and 146 non-substrates was compiled from literature. Random sampling was applied to split such dataset into a 125-compound training set and a 125compound test set. Linear discriminant analysis was conducted to select conformation-independent models from different subsets of Dragon descriptors. Later, simple data fusion schemes were used to combine individual models in order to optimize specificity. Receiving Operating Characteristic curves were applied to compare model performance and to select the best data fusion scheme. The ensemble showed $90 \%$ accuracy in the classification of the substrates, though of course a different balance between sensitivity and specificity could be easily achieved by selecting a different score threshold. Cascade application of the preceding ensemble together with structure-based approaches and a ligand-based classificatory model capable of identifying drugs with anticonvulsant effects in the MES test (see next section for details on the molecular docking) in the virtual screening campaign of ZINC and Drugbank databases led to the identification of anticonvulsant compounds predicted as non-substrates for Pgp [76]. These in silico filters were also applied to an in house library of anticonvulsant compounds previously reported by the same group, including antimicrobial Propylparaben (compound VII) and non-nutritive sweetener potassium Acesulfame (compound VIII) [77, 78]. The anticonvulsants discovered through this protocol are displayed in (Fig. 4).

The same group has recently reported linear model ensemble capable of identifying substrates for wild-type human ABCG2 [74, 79], which might well be integrated to the previously described in silico filters.

\subsection{Structure-based Approaches}

Structure-based approaches are valuable tools in drug design, since they provide atomic details on the interactions between the target and ligands. Particularly, docking simulations propose possible binding geometries of the complexes, and quantify in some way their binding energies through their scoring functions. This information allows the structural optimization of the ligands to improve their interactions with the targets (or to avoid them in antitargets). Additionally, docking scores provide a numerical variable to discriminate between binders and non-binders (of a defined target) in structure-based virtual screening campaigns.
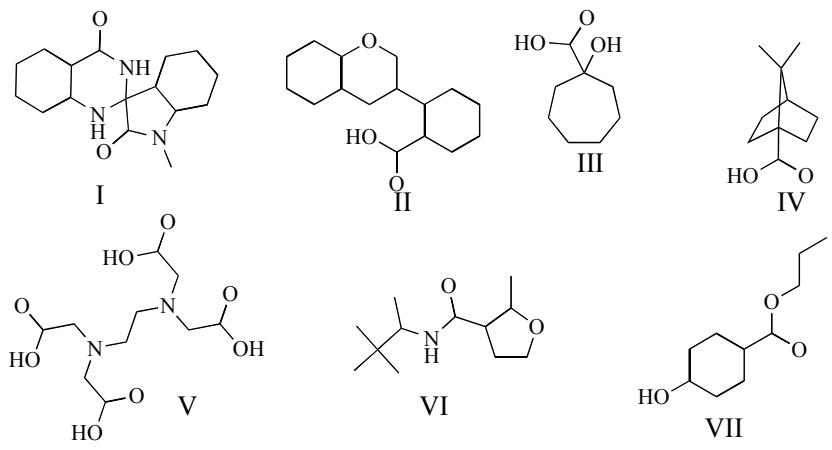

$$
\underbrace{\mathrm{O}_{\mathrm{O}}^{\mathrm{O}} \mathrm{S}^{\prime}}_{\text {VIII }}
$$
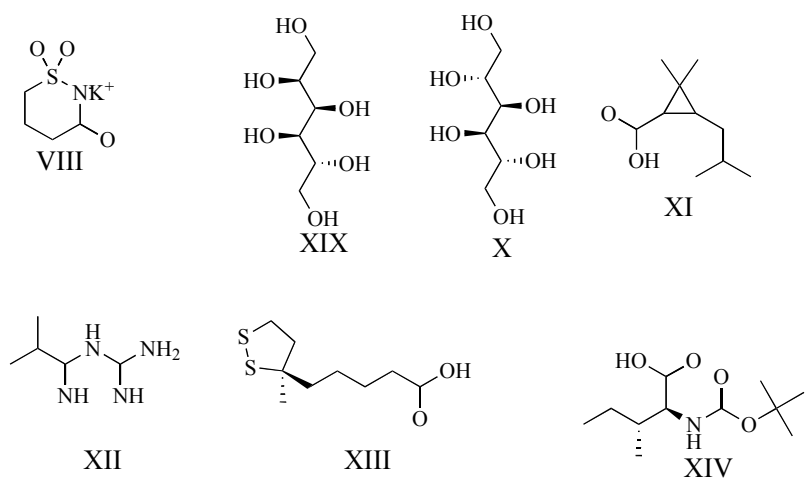

Fig. (4). Anticonvulsant drug candidates predicted as non-substrates of P-gp by joint application of structure- and ligand-based approaches.

Regarding P-gp, structure-based methods deal with the lack of experimental information about the 3D architecture of the human protein. Therefore, the target is usually modeled by comparative analysis (homology modeling techniques) with templates of mouse P-gp, which shares more than $80 \%$ of sequence identity with human P-gp [79]. The glycoprotein is composed by two sets of transmembrane segments (TMs 1-3,6,10,11 and TMs 4,5,7-9,12), which generate an internal cavity that contains multiple binding sites (Fig. 5) [80 and refs therein]. Experimental data has proved the capacity of P-gp to interact at the same time with more than one substrate, and the existence of new potential sites of interaction with small molecules on the exterior of Pgp cavity [80]. This information is employed in docking protocols to find P-gp substrates/inhibitors. They have, in general, less accuracy than ligand-based approaches $[81,75$, 82]. However, there are successful examples in relation to the identification of P-gp substrates/inhibitors by means of docking simulations, and some of them provide information about the conformations of the binders in the active site [79 and refs therein].

To exploit the potentialities of both ligand- and targetbased methods, docking-based filter was coupled a to a ligand based search of anticonvulsant compounds with no Pgp interactions [76]. As mentioned before, a ligand-based model ensemble was initially applied on the ZINC and Drug Bank databases, with the aim of identifying new anticonvulsant predicted as non-substrates of P-gp. The best 380 candidates were then submitted to the docking simulations and the compounds presumed with high interaction with the glycoprotein were discarded. 
The candidates were docked into a homology model of human P-gp based on the mouse P-gp structure as template (PDB code: 3G61) [80]. Several scoring functions and conditions were analyzed to select the best model. The abilities of the simulations to reproduce experimental data as well to discriminate known P-gp binders from non-binders were tested. A flexible receptor model with the scoring function of Autodock Vina was able to reproduce experimental conformations of mouse P-gp complexes as well to predict the $85 \%$ of the binders and the $77 \%$ of nonbinders [80]. Fig. (5) shows the docking solution for the binding of Saquinavir to the P-gp active site, as an example of the characteristic interactions predicted for known binders. The active sites into the P-gp cavity are mostly hydrophobic and they are composed by residues with lipophilic or aromatic side chains.

From the 380 candidates selected by ligand-based screening, 275 structures were considered as non-binders by docking; evidencing a high level of consensus between both protocols. As mentioned before, some of the anticonvulsants identified with sequential screening are shown in Fig. (4).

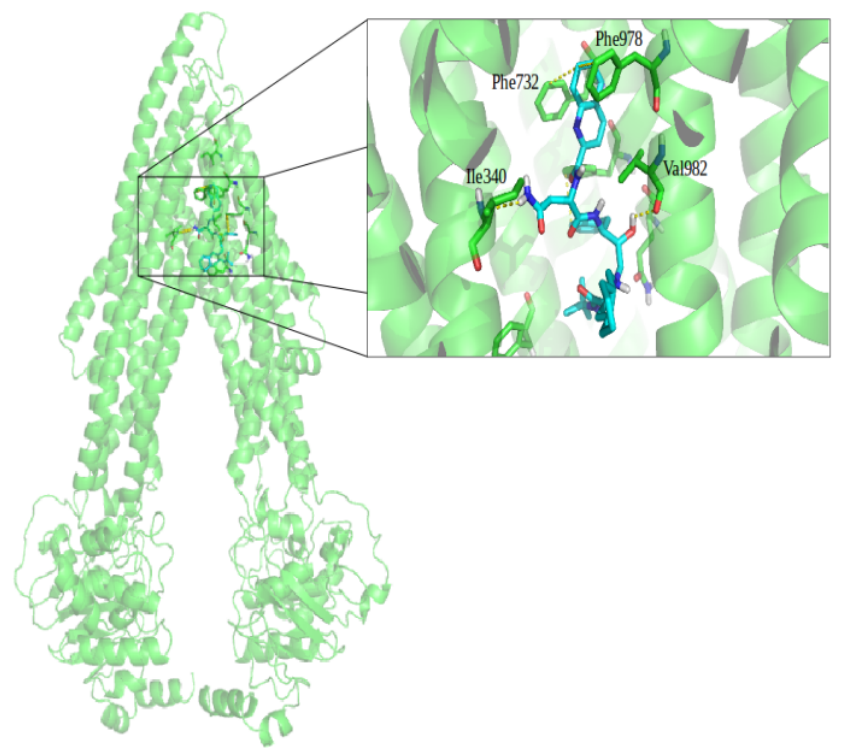

Fig. (5). Structure of the model of human P-gp colored in green with Saquinavir in its predicted binding conformation. Residues of the binding pocket are highlighted in green. Carbon atoms of Saquinavir are highlighted in cyan.

\section{IN VITRO EXPERIMENTAL VALIDATION OF THE PREDICTIONS}

Parent and MDR1 transfected Madin-Darby canine kidney epitelial cells were obtained from the Netherlands Cancer Institute (Amsterdam, The Netherlands). Cells were grown in $25-\mathrm{cm}^{2}$ culture flasks using DMEM with $10 \%$ fetal bovine serum, 1\% L-glutamine, $1 \%$ non-essential amino acids and penicillin and streptomycin at $37^{\circ} \mathrm{C}$ in $5 \% \mathrm{CO}_{2}$ atmosphere. Cells were split twice a week at 70 to $80 \%$ confluence in a ratio of 1:20 or 1:30 using s Trypsin-EDTA solution $(0.25 \%)$. All transport assays were done with cells from passages 19 to 43 . Cells were kept at $37^{\circ} \mathrm{C}$ in $5 \% \mathrm{CO}_{2}$.

The cells were seeded in 6-well Costar Snapwell plates with polycarbonate membrane inserts at a density of 50,000 cells per insert $\left(1.12 \mathrm{~cm}^{2}\right)$ and grown for 4 days in culture medium. The medium was replacing every day. The apical media volume was $0.5 \mathrm{ml}$, and the basal volume was $2 \mathrm{ml}$. Integrity of the cell monolayers was determined by measuring the trans-epithelial electrical resistance (TEER, $\Omega . \mathrm{cm} 2$ ) using an epithelial voltammeter (Millicell-ERS; Millipore Corporation). In addition, the integrity was checked using Atenolol (ATOL). The apparent permeability coefficients (Papp) of Atenolol across MDCK II- MDR I cell monolayers were typically $1-5 \times 10^{-7} \mathrm{~cm} / \mathrm{s}$. The expression of Pgp was checked by Western Blot analysis and by transports assay with Trimethoprim, a substrate for Pgp [83].

On the day of the experiment, culture medium was removed, and cells were washed three times with media transport (HBSS, Hanks' balance salt solution, $\mathrm{pH}$ 7.4, Gibco-BRL). The filter inserts containing the cell monolayers were placed in an Ussing chamber, and were maintained at $37^{\circ} \mathrm{C}$ and under constant gassing with carbogen. Test compounds were added to the donor side (4 $\mathrm{ml}$ for the apical and basal chamber). At 20, 40, 60, 80, 100 and $120 \mathrm{~min}$, samples $(400 \mu \mathrm{l})$ were taken from receiver compartment followed by addition of $400 \mu \mathrm{l}$ of transport media. For the inhibition experiments, cell monolayers were incubated with Amiodarone Chlorhydrate $(50 \mu \mathrm{M})$ [84] for 1 $\mathrm{h}$ in apical and basolateral chambers before adding the test compound.

Efflux of Trimethoprim (TMP, $100 \mu \mathrm{M}$ ), Compound VIII (Acesulfame, $100 \mu \mathrm{M}$ ), Compound VII (Propylparabene, $100 \mu \mathrm{M})$, Compound II (2-(2-oxo-2Hchromen-3-yl)benzoic acid, $2.5 \mu \mathrm{M}$ ) and Compound I (1methyl-1,2,3,4'-tetrahidro-1'H-spiro[indol-3,2'-quinazolin]-

2,4'-dione, $1 \mu \mathrm{M}$ ) across the confluent monolayer of cells was measured in both directions, i.e., apical to basolateral (A-B) and basolateral to apical (B-A) in the presence and absence of Amiodarone. Test drugs were dissolved in buffer media or propylene glycol (final concentration of $3.5 \%$ solvent). Apparent permeability (Papp) was calculated with the equation Papp $=1 / \mathrm{AC0}(\mathrm{dQ} / \mathrm{dt})$, where $\mathrm{A}$ is membrane surface area $\left(1.12 \mathrm{~cm}^{2}\right), \mathrm{C} 0$ is the initial concentration in the donor compartment at $\mathrm{t}=0$, and $\mathrm{dQ} / \mathrm{dt}$ is amount of drug transported within a given time period. The net efflux (ER) of a test compound was assessed by calculating the ratio of Papp in the B-A direction versus Papp in the A-B direction. $\mathrm{ER}=\mathrm{Papp} \mathrm{B} \rightarrow \mathrm{A} / \mathrm{Papp} \mathrm{A} \rightarrow \mathrm{B}$.

For Pgp substrates, the absorption should be decreased and the secretion increased in cell lines over-expressing Pgp [85]. In the presence of a specific inhibitor for Pgp, the $\mathrm{ER} \approx 1$.

The samples were quantified by HPLC system: Dionex Ultimate 3000 UHPLC (Thermo Scientific, Sunnyvale, CA) configured with a dual gradient tertiary pump (DGP-3000) and a DAD-3000 diode array detector. The stationary phase was a Phenomenex Luna RP-18 column $(150$ x $4 \mathrm{~mm}, 5 \mu \mathrm{m})$ and the mobile phase was: ATOL: $\mathrm{H}_{3} \mathrm{PO}_{4} 0.1 \%$ : Methanol 


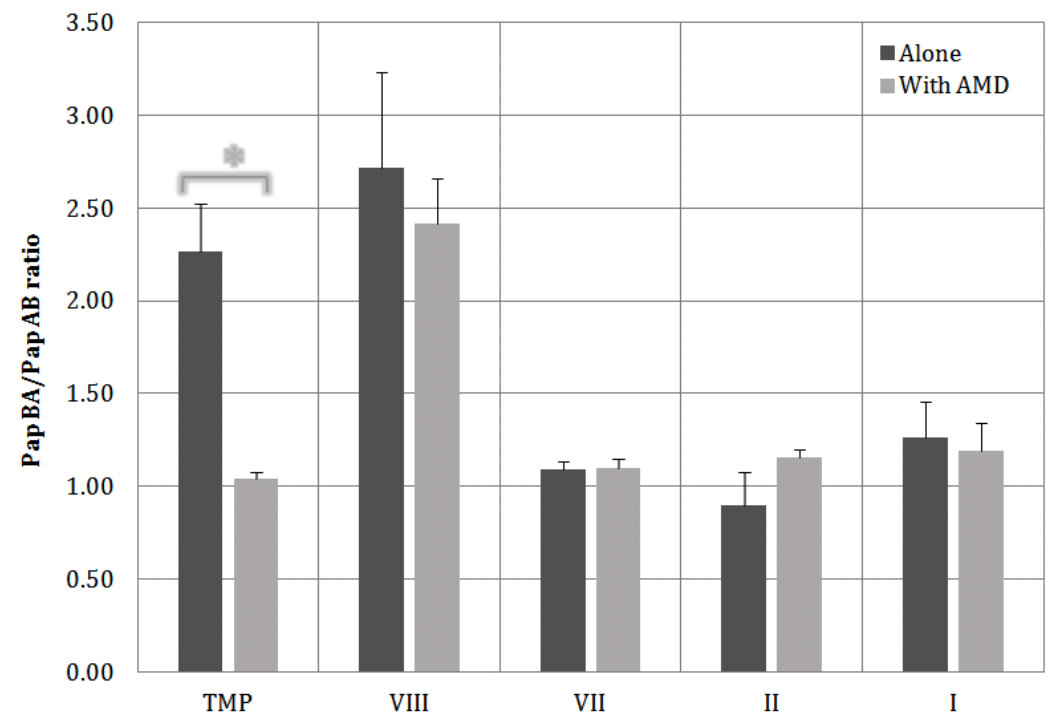

Fig. (6). Permeability ratios (B-A/A-P) of the candidate anticonvulsant drugs mentioned.

$80: 20$, the flow rate was set at $1 \mathrm{~mL} / \mathrm{min}$ and detection was performed at 225 and $274 \mathrm{~nm}$; TMP: $\mathrm{H}_{3} \mathrm{PO}_{4}$ 0.1\%: Methanol $65: 35$, the flow rate was set at $1.3 \mathrm{~mL} / \mathrm{min}$ and detection was performed at 230 and $270 \mathrm{~nm}$; Compound VIII: Buffer $\mathrm{KH}_{2} \mathrm{PO}_{4} 20 \mathrm{mM}$, adjusted to $\mathrm{pH} 2.5$ with $\mathrm{H}_{3} \mathrm{PO}_{4}$ : Methanol $80: 20$, the flow rate was set at $1 \mathrm{~mL} / \mathrm{min}$ and detection was performed at 230 and $240 \mathrm{~nm}$; Compound VII: $\mathrm{H}_{3} \mathrm{PO}_{4} 0.1 \%$ : Methanol 35:65, the flow rate was set at $1.2 \mathrm{~mL} / \mathrm{min}$ and detection was performed at 230 and $250 \mathrm{~nm}$; Compound II: Buffer $\mathrm{KH}_{2} \mathrm{PO}_{4} 20 \mathrm{mM}$, adjusted to $\mathrm{pH} 2.5$ with $\mathrm{H}_{3} \mathrm{PO}_{4}$ : Methanol 35:65, the flow rate was set at $1 \mathrm{~mL} / \mathrm{min}$ and detection was performed at $220 \mathrm{~nm}$; Compound I: Buffer $\mathrm{KH}_{2} \mathrm{PO}_{4} 20 \mathrm{mM}$, adjusted to $\mathrm{pH} 2.5$ with $\mathrm{H}_{3} \mathrm{PO}_{4}$ : Methanol $30: 70$, the flow rate was set at $1 \mathrm{~mL} / \mathrm{min}$ and detection was performed at $297 \mathrm{~nm}$.

Student's $\mathrm{T}$ test for two samples assuming equal variances was conducted for statistical comparisons.

The values obtained with TMP indicated that the ER value is 2.5 times higher in the absence of inhibitor than in its presence, demonstrating expression of Pgp. None of the four drugs evaluated showed significant differences when calculating the ER in presence and absence of Amiodarone (Fig. 6), indicating that in the experimental conditions studied P-gp efflux does not influence the transport of these drugs, which seems consistent with the predictions of the models. Nevertheless, it should be noted that compound VIII presents an ER significantly different from 1, thus suggesting it might be recognized by other efflux transporters different than P-gp, which illustrates the importance of complementing P-gp models with other models to identify efflux by different ABC transporters.

\section{CONCLUSIONS}

Though it is unlikely that a single mechanistic hypothesis will account for a very complex phenomenon such as multidrug resistance, the body of preclinical, clinical and pharmacogenomics evidence suggest a role of $\mathrm{ABC}$ transporters in refractory epilepsy. Most of the studies so far have focused on the potential role of P-gp in drug resistant epilepsy; while initial studies have disregarded the effect of other ABC transporters in epilepsy, such studies should be reexamined at the light of recent advances in the field, including more recent and complex in vitro and in vivo models (e.g. the CETA assay and double and triple knockout animal models, which may contribute to explore, respectively, the influence of passive diffusion on drug permeability and the cooperative function of $\mathrm{ABC}$ pumps). Despite some existing AEDs are not likely to be transported by $\mathrm{ABC}$ carriers, current clinical criteria to diagnose refractory epilepsy does not actually exclude the possibility that a refractory patient could respond to available medications: in absence of definitive clinical validation of the transporter hypothesis, standard management of epilepsy does not consider whether previously administered (and unsuccessful) drugs are or are not substrates of $\mathrm{ABC}$ transporters. Nor does it contemplate assessment of the ABC transporters function in a particular patient.

In relation to novel therapeutic approaches to drug resistant epilepsy, the use of nano-pharmaceutical delivery systems and the design of new AEDs which are not recognized by $\mathrm{ABC}$ transporters (an antitarget approximation) represent safer options compared to co-administration of ABC transporters inhibitors. These are still, however, underexplored alternatives for the management of refractory epilepsy. We have discussed a screening protocol based on the antitarget approach, which integrates in silico, in vitro and in vivo tools to select AED candidates oriented to the therapy of drug resistant epilepsy linked to upregulation of $\mathrm{ABC}$ transporters.

\section{CONFLICT OF INTEREST}

The author(s) confirm that this article content has no conflict of interest. 


\section{ACKNOWLEDGEMENTS}

MC and GP thanks INAME, ANMAT. MDI, AVE, PP, LG and AT thank CONICET.

\section{AT thanks ANPCyT PICT 2010-2531 and CONICET PIP.}

LBB thanks Incentivos UNLP and CONICET PIP.

\section{REFERENCES}

[1] World Health Organization. Fact sheet number 999: Epilepsy. http://www.who.int/mediacentre/factsheets/fs999/en/ (Update May, 2015; Accessed August 19, 2015).

[2] Brodie, M.J.; Barry, S.J.; Bamagous, G.A.; Norrie, J.D.; Kwan, P. Patterns of treatment response in newly diagnosed epilepsy. Neurology, 2012, 78(20), 1548-1554.

[3] Sinha, S.; Siddiqui, K.A. Definition of intractable epilepsy. Neurosciences (Riyadh), 2011, 16(1), 3-9.

[4] Löscher, W.; Schmidt, D. Modern antiepileptic drug development has failed to deliver: ways out of the current dilemma. Epilepsia, 2011, 52(4), 657-578.

[5] Löscher, W.; Klitgaard, H.; Twyman, R. E.; Schmidt, D. New avenues for anti-epileptic drug discovery and development. Nat. Rev. Drug. Discov., 2013, 12(10), 757-776.

[6] Kwan, P.; Brodie, M. J. Potential role of drug transporters in the pathogenesis of medically intractable epilepsy. Epilepsia, 2005, 46(2), 224-235.

[7] Remy, S.; Beck, H. Molecular and cellular mechanisms of pharmacoresistance in epilepsy. Brain, 2006, 129(Pt 1), 18-35.

[8] Schmidt, D.; Löscher, W. Drug resistance in epilepsy: putative neurobiologic and clinical mechanisms. Epilepsia, 2005, 46(6), 858-877.

[9] Fang, M.; Xi, Z. Q.; Wu, Y.; Wang, X. F. A new hypothesis of drug refractory epilepsy: neural network hypothesis. Med. Hypotheses, 2011, 76(6), 871-876.

[10] Löscher, W.; Klotz, U.; Zimprich, F.; Schmidt, D. The clinical impact of pharmacogenetics on the treatment of epilepsy. Epilepsia, 2009, 50(1), 1-23.

[11] Rogawski, M. A.; Johnson, M. R. Intrinsic severity as a determinant of antiepileptic drug refractoriness. Epilepsy Curr., 2008, 8(5), 127-130

[12] Schmidt, D.; Löscher, W. New developments in antiepileptic drug resistance: an integrative view. Epilepsy Curr., 2009, 9(2), 47-52.

[13] Kobow, K.; El-Osta, A.; Blumcke, I. The methylation hypothesis of pharmacoresistance in epilepsy. Epilepsia, 2013, 54(Suppl. S2), 4147.

[14] Wiebe, S.; Jette, N. Pharmacoresistance and the role of surgery in difficult to treat epilepsy. Nar. Rev. Neurol., 2012, 8(12), 669-677.

[15] Rogawski, M. A. The intrinsic severity hypothesis of pharmacoresistance to antiepileptic drugs. Epilepsia, 2013, 54(Suppl 2), 33-40.

[16] Talevi, A.; Bruno-Blanch, L.E. In: Pharmacaoresistance in Epilepsy. From Genes and Molecules to Promising Therapies; Rocha and Cavalheiro, Eds.; Springer: New York, 2012, pp. 207224.

[17] Rees, D. C.; Johnson, E.; Lewinson, O. ABC transporters: the power to change. Nat. Rev. Mol. Cell Biol., 2009, 10(3), 218-227.

[18] Talevi, A.; Bruno-Blanch, L.E. In: The Blood-Brain Barrier: New Research; Montenegro and Juárez, Eds.; Nova Science Publishers: New York, 2012, pp. 117-144.

[19] Glavinas, H.; Krajscsi, P.; Cserepes, J.; Sarkadi, B. The role of $\mathrm{ABC}$ transporters in drug resistance, metabolism and toxicity. Curr. Drug Deliv., 2004, 1(1), 27-42.

[20] Abuznait, A. H.; Kaddoumi, A. Role of ABC transporters in the pathogenesis of Alzheimer's disease. ACS Chem. Neurosci., 2012, 3(11), 820-831.

[21] Potschka, H.; Fedrowitz, M.; Löscher, W. P-glycoprotein and multidrug resistance-associated protein are involved in the regulation of extracellular levels of the major antiepileptic drug carbamazepine in the brain. NeuroReport, 2001, 12(16), 35573560.

[22] Potschka, H.; Fedrowitz, M.; Löscher, W. Multidrug resistance protein MRP2 contributes to blood-brain barrier function and restricts antiepileptic drug activity. J. Pharmacol. Exp. Ther., 2003, 306(1), 124-131.

[23] Clinckers, R.; Smolders, I.; Meurs, A.; Ebinger, G.; Michotte, Y. Quantitative in vivo microdialysis study on the influence of multidrug transporters on the blood-brain barrier passage of oxcarbazepine: concomitant use of hippocampal monoamines as pharmacodynamics markers for the anticonvulsant activity. $J$. Pharmacol. Exp. Ther., 2005, 314(2), 725-731.

[24] van Vliet, E. A.; van Schaik, R.; Edelbroek, P. M.; Redeker, S. Aronica, E.; Wadman, W. J.; Marchi, N.; Vezzani, A.; Gorter, J. A. Inhibition of the multidrug transporter P-glycoprotein improves seizure control in phenytoin-treated chronic epileptic rats. Epilepsia, 2006, 47(4), 672-680.

[25] Brandt, C.; Bethmann, K.; Gastens, A. M.; Löscher, W. The multidrug transporter hypothesis of drug resistance in epilepsy: proof-of-principle in a rat model of temporal lobe epilepsy. Neurobiol. Dis., 2006, 24(1), 202-211.

[26] Höcht, C.; Lazarowski, A.; González, N. N.; Auzmendi, J.; Opezzo, J. A.; Bramuglia, G. F.; Taira, C. A.; Girardi, E. Nimodipine restores the altered hippocampal phenytoin pharmacokinetcs in a refractory epileptic model. Neurosci. Lett., 2007, 413(2), 168-172.

[27] Jambroszyk, M.; Tipold, A.; Potschka, H. Add-on treatment with verapamil in pharmacoresistant canine epilepsy. Epilepsia, 2011, 52(2), 284-291.

[28] Tishler, D. M.; Weinberg, K. I.; Hinton, D. R.; Barbaro, N.; Annett, G. M.; Raffel, C. MDR1 gene expression in brain of patients with medically intractable epilepsy. Epilepsia, 1995, 36(1), 1-6.

[29] Dombrowski, S. W.; Desai, S. Y.; Marroni, M.; Cucullo, L.; Goodrich, K.; Bingaman, W.; Mayberg, M. R.; Bengez, L.; Janigro, D. Overexpression of multidrug resistance genes in endothelial cells from patients with refractory epilepsy. Epilepsia, 2001, 42(12), 1501-1506.

[30] Sisodiya, S. M.; Lin, W. R.; Harding, B. N.; Squier, M. V.; Thosn, W. Drug resistance in epilepsy: expression of drug resistance proteins in common causes of refractory epilepsy. Brain, 2002, 125(Pt. 1), 22-31.

[31] Aronica, E.; Gorter, J. A.; Jansen, G. H.; van Veelen, C. W.; van Rijen, P. C.; Leenstra, S.; Ramkema, M.; Scheffer, G. L.; Scheper, R. J.; Troost, D. Expression and cellular distribution of multidrug transporter proteins in two major causes of medically intractable epilepsy: focal cortical dysplasia and glioneuronal tumors. Neuroscience, 2003, 118(2), 417-429.

[32] Aronica, E.; Gorter, J. A.; Redeker, S.; van Vliet, E. A.; Ramkema, M.; Scheffer, G. L.; Scheper, R. J.; van der Valk, P.; Leenstra, S.; Baayen, J. C.; Spliet, W. G.; Troost, D. Localization of breast cancer resistance protein (BCRP) in microvessel endothelium of human control and epileptic brain. Epilepsia, 2005, 46(6), 849857.

[33] Lazawoski, A.; Massaro, M.; Schteinschnaider, A.; Intruvini, S ; Sevlever, G.; Rabinowicz, A. Neuronal MDR-1 gene expression and persistent low levels of anticonvulsants in a child with refractory epilepsy. Ther. Drug Monit., 2004, 26(1), 44-46.

[34] Kubota, H.; Ishihara, H.; Langmann, T.; Schmitz, G.; Stieger, B.; Wieser, H. G.; Yonekawa, Y.; Frei, K. Distribution and functional activity of $\mathrm{P}$-glycoprotein and multidrug resistance-associated proteins in human brain microvascular endothelial cells in hippocampal sclerosis. Epilepsy Res., 2006, 68(3), 213-228.

[35] Ak, H.; Ay, B.; Tanriverdi, T.; Sanus, G. Z.; Is, M.; Sar, M.; Oz, B.; Ozkara, C.; Ozyurt, E.; Uzan, M. Expression and cellular distribution of multidrug resistance-related proteins in patients with focal cortical dysplasia. Seizure, 2007, 16(6), 493-503.

[36] Feldmann, M.; Asselin, M. C.; Liu, J.; Wang, S.; McMahon, A.; Anton-Rodriguez, J.; Walker, M.; Symms, M.; Brown, G.; Hinz, R.; Matthews, J.; Bauer, M.; Langer, O.; Thom, M.; Jones, T.; Vollmar, C.; Duncan, J. S; Sisodiya, S. M.; Koepp, M. J. Pglycoprotein expression and function en patients with temporal lobe epilepsy: a case-control study. Lancet Neurol., 2013, 12(8), 777-785

[37] Feldmann, M.; Koepp, M. P-glycoprotein imaging in temporal lobe epilepsy: in vivo PET experiments with the Pgp substrate [11C]verapamil. Epilepsia, 2012, 53(Suppl. 6), 60-63.

[38] Löscher, W.; Langer, O. Imaging of P-glycoprotein function and expression to elucidate mechanisms of pharmacoresistance in epilepsy. Curr. Top. Med. Chem., 2010, 10(17), 1785-1791. 
[39] Potschka, H.; Luna-Munguia, H. CNS transporters and drug delivery in epilepsy. Curr. Pharm. Des., 2014, 20(10), 1534-1542.

[40] Summers, M. A.; Moore, J. L.; McAuley, J. W. Use of verapamil as a potential P-glycoprotein inhibitor in a patient with refractory epilepsy. Ann. Pharmacother., 2004, 38(6), 1631-1634.

[41] Ianetti, P.; Spalice, A.; Parisi, P. Calcium-channel blocker verapamil administration in prolonged and refractory status epilepticus. Epilepsia, 2005, 46(6), 967-969.

[42] Schmitt, F. C.; Dehnicke, C.; Merschhemke, M.; Meencke, H. J. Verapamil attenuates the malignant treatment course in recurrent status epilepticus. Epilepsy Behav., 2010, 17(4), 565-568.

[43] Pirker, S.; Baumgartner, C. Termination of refractory focal status epilepticus by the P-glycoprotein inhibitor verapamil. Eur. J. Neurol., 2011, $18(12)$, e151.

[44] Nicita. F.; Spalice, A.; Papetti, L.; Nikanorova, M.; Ianetti, P.; Parisi, P. Efficacy of verapamile as an adjunctive treatment in children with drug-resistant epilepsy: A pilot study. Seizure, 2014, 23(1), 36-40.

[45] Borlot, F.; Wither, R. G.; Ali, A.; Wu, N.; Verocai, F.; Andrade, D. M. A pilot double-blind trial using verapamil as adjuvant therapy for refractory seizures. Epilepsy Res., 2014, 108(9), 1642-1651.

[46] Haerian, B.S.; Lim, K.S.; Tan, C.T.; Raymond, A.A.; Mohamed, Z. Association of $\mathrm{ABCB} 1$ gene polymorphisms and their haplotypes with response to antiepileptic drugs: a systematic review and metaanalysis. Pharmacogenomics, 2011, 12(5), 713-725.

[47] Yu, L.; Liao, W. P.; Yi, Y. H.; Qiu, G. ABCB1 G2677T/A polymorphism is associated with the risk of drug-resistant epilepsy in Asians. Epilepsy Res., 2015, 115, 100-108.

[48] Lv, W. P.; Han, R. F.; Shu, Z. R. Associations between the C3435T polymorphism of the $\mathrm{ABCB} 1$ gene and drug resistance in epilepsy: a meta-analysis. Int. J. Clin. Exp. Med., 2014, 7(11), 3924-3932.

[49] Li, S. X.; Liu, Y. Y.; Wang, Q. B. ABCB1 gene C3435T polymorphim and drug resistance in epilepsy: evidence based on 8,604 subjects. Med. Sci. Monit., 2015, 21, 861-868.

[50] Zhang, C.; Kwan, P.; Zuo, Z.; Baum, L. The transport of antiepileptic drugs by P-gp. Adv. Drug. Deliv. Rev., 2012, 64(10), 930-942.

[51] Löscher, W.; Luna-Tortós, C.; Römermann, K.; Fedrowitz, M. Do ATP-binding cassette transporters cause pharmacoresistance in epilepsy? Problems and approaches in determining which antiepileptic drugs are affected. Curr. Pharm. Des., 2011, 17(26), 2808-2828.

[52] Luna-Tortós, C.; Fedrowitz, M.; L[oscher, W. Several major antiepileptic drugs are substrates for human P-glycoprotein. Neuropharmacology, 2008, 55(8), 1364-1375.

[53] Löscher, W.; Luna-Tortós, C.; Römermann, K.; Fedrowitz, M. Do ATP-binding cassette transporters cause pharmacoresistance in epilepsy? Problems and approaches in determining which antiepileptic drugs are affected. Curr. Pharm. Des., 2011, 17(26), 2808-2828.

[54] Cerveny, L.; Pavek, P.; Malakova, J.; Staud, F.; Fendrich, Z. Lack of interactions between breast cancer resistance protein (bcrp/abcg2) and selected antiepileptic agents. Epilepsia, 2006, 47(3), 461-468.

[55] Römermann, K.; Helmer, R.; Löscher, W. The antiepileptic drug lamotrigine is a substrate of mouse and human breast cancer resistance protein (ABCG2). Neuropharmacology, 2015, 93, 7-14.

[56] Nakanishi, H.; Yonezawa, A.; Matsubara, K.; Yano, I. Impact of Pglycoprotein and breast cancer resistance protein on the brain distribution of antiepileptic drugs in knockout mouse models. Eur. J. Pharmacol., 2013, 710(1-3), 20-8.

[57] Shawahna, R:; Uchida, Y.; Declèves, X.; Ohtsuki, S.; Yousif, S.; Dauchy, S.; Jacob, A.; Chassoux, F.; Daumas-Duport, C.; Couraud, P. O.; Terasaki, T.; Chermann, J. M. Transcriptomic and quantitative proteomic analysis of transporters and drug metabolizing enzymes in freshlyisolated human brain microvessels. Mol. Pharm., 2011, 8(4), 1332-1341.

[58] Uchida, Y.; Ohtsuki, S.; Katsukura, Y.; Ikeda, C.; Suzuki, T.; Kamiie, J.; Terasaki, T. Quantitative targeted absolute proteomics of human blood-brain barrier transporters and receptors. $J$. Neurochem., 2011, 117(2), 333-345.

[59] Lin, F.; Marchetti, S.; Pluim, D.; Iusuf, D.; Mazzanti, R.; Chellens, J. H.; Beijnen, J. H.; van Tellingen, O. Abcc4 together with abcb1 and abcg2 form a robust cooperative drug efflux system that restricts the brain entry of camptothecin analogues. Clin. Cancer Res., 2013, 19(8), 2084-2095.

[60] Tiwari, A. K.; Zhang, R.; Gallo, J. M. Overlapping functions of $\mathrm{ABC}$ transporters in topotecan disposition as determined in gene knockout mouse models. Mol. Cancer. Ther., 2013, 12(7), 13431355.

[61] Vlaming, M. L.; Pala, Z.; van Esch, A.; Wagenaar, E.; de Waart, D. R.; van de Wetering, K.; van der Kruijssen, C. M.; Oude Elferink, R. P.; van Tellingen, O.; Schinkel, A. H. Functionally overlapping roles of Abcg2 (Bcrp1) and Abcc2 (Mrp2) in the elimination of methotrexate and its main toxic metabolite 7-hydroxymethotrexate in vivo. Clin. Cancer Res., 2009, 15(9), 3084-3093.

[62] Potschka, H.; Luna-Munguia, H. CNS transporters and drug delivery in epilepsy. Curr. Pharm. Des. 2014, 20, 1435-1542.

[63] Cirrito, J. R.; Deane, R.; Fagan, A. M.; Spinner, M. L.; Parsadanian, M.; Finn, M. B.; Jiang, H.; Prior, J. L.; Sagare, A.; Bales, K. R.; Paul, S. M.; Zlokovic, B. V.; Piwnica-Worms, D.; Holtzman, D. M. P-glycoprotein deficiency at the blood-brain barrier increases amyloid-beta deposition in an Alzheimer disease mouse model. J. Clin. Invest., 2005, 115(11), 3285-3290.

[64] Bartels, A. L.; Willemsen, A. T.; Kortekaas, R.; de Jong, B. M.; de Vries, R.; de Klerk, O.; van Oostrom, J. C.; Portman, A.; Leenders, K. L. Decreased blood-brain barrier $P$-glycoprotein function in the progression of Parkinson's disease, PSP and MSA. J. Neural. Transm., 2009, 5(11), 1349-1358.

[65] Vautier, S.; Fernandez, C. ABCB1: the role in Parkinson's disease and pharmacokinetics of antiparkinsonian drugs. Expert Opin. Drug Metab. Toxicol., 2009, 5(11), 1349-1358.

[66] Potschka, H. Role of CNS efflux drug transporters in antiepileptic drug delivery: Overcoming CNS efflux drug transport. Adv. Drug Deliv Rev,. 2012, 64(10), 943-952.

[67] Rosillo-de la Torre, A.; Luna-Bárcenas, G.; Orozco-Suárez, S.; Salgado-Ceballos, H.; García, P.; Lazarowski, A.; Rocha, L. Pharmacoresistant epilepsy and nanotechnology. Front. Biosci. (Elite Ed), 2014, 6, 329-340

[68] Bennewitz, M. F.; Saltzman, W. M. Nanotechnology for delivery of drugs to the brain for epilepsy. Nanotherapeutics, 2009, 6, 323336.

[69] Demel, M. A.; Schwaha, R.; Krämer, O.; Ettmayer, P.; Haaksma, E. E. J.; Ecker, G. In silico prediction of substrate properties for ABC-multidrug transporters. Expert Opin. Drug Metab. Toxicol, 2008, 4(9), 1167-1180.

[70] Ecker, G. F.; Stockner, T.; Chiba, P. Computational models for prediction of interactions with ABC-transporters. Drug Discov. Today, 2008, 13(7/8), 311-317.

[71] Chen, L.; Li, Y.; Yu, H.; Zhang, L.; Hou, T. Computational models for predicting substrates or inhibitors of P-glycoprotein. Drug Discov. Today, 2012, 17(7/8), 343-351.

[72] Pinto, M.; Digles, D.; Ecker, G. F. Computational models for predicting the interaction with $\mathrm{ABC}$ transporters. Drug Discov. Today Technol., 2014, 12, e69-e77.

[73] Hazai, E.; Hazai, I.; Ragueneau-Majlessi, I.; Chung, S. P.; Bikadi, Z.; Mao, Q. Predicting substrates of the human breast cancer resistance protein using a support vector machine method. $B M C$ Bioinformatics, 2013, 14, 130.

[74] Gantner, M. E.; Di Ianni, M. E.; Ruiz, M. E.; Talevi, A.; BrunoBlanch, L. E. Development of conformation independent computational models for the early recognition of Breast Cancer Resistance Protein substrates. Biomed Res. Int., 2013, 2013, article 863592 .

[75] Di Ianni, M.; Talevi, A.; Castro, E. A.; Bruno-Blanch, L. E. Development of a highly specific ensemble of topological models for early identification of P-glycoprotein substrates. J. Chemometr., 2011, 6, 313-322.

[76] Di Ianni, M. E.; Enrique, A. V.; Palestro, P. H.; Gavernet, L.; Talevi, A.; Bruno-Blanch, L. E. Several new diverse anticonvulsant agents discovered in a virtual screening campaign aimed at novel antiepileptic drugs to treat refrctory epilepsy. J. Chem. Inf. Model., 2012, 52(12), 3325-3330.

[77] Talevi, A.; Bellera, C. L.; Castro, E. A.; Bruno-Blanch, L. E. A successful virtual screening application: Prediction of anticonvulsant activity in MES test of widely used pharmaceutical and food preservatives methylparaben and propylparaben. $J$. Comput. Aided Mol. Des., 2007, 21(9), 527-538. 
[78] Talevi, A.; Enrique, A. V.; Bruno-Blanch, L. E. Anticonvulsant activity of artificial sweeteners: A structural link between sweettaste receptor T1R3 and brain glutamate receptors. Bioorg. Med. Chem. Lett., 2012, 22(12), 4072-4074.

[79] Gantner, M. E.; Alberca, L. N.; Mercader, A. G.; Bruno-Blanch, L. E.; Talevi, A. Application of the Enhanced Replacement Method and ensemble learning for the prediction of BCRP/ABCG2 substrates. Curr. Bioinform. In press.

[80] Palestro P. H.; Gavernet L.; Estiu G. L.; Bruno Blanch L. E. Docking Applied to the Prediction of the Affinity of Compounds to P-Glycoprotein. BioMed research international, 2014, Article ID 358425

[81] Szewczyk P.; Tao H.; McGrath A. P.; Villaluz M.; Rees S.; Lee S. C.; Doshi R.; Urbatsch I. L.; Zhang Q.; Chang G.. Snapshots of ligand entry, malleable binding and induced helical movement in Pglycoprotein. Acta Crystallographica Section D: Biological Crystallography, 2015, 71(Pt 3):732-741
[82] Klepsch F.; Vasanthanathan P.; Ecker G. F. Ligand and structurebased classification models for prediction of P-glycoprotein inhibitors. Journal of chemical information and modeling, (2012) 54(1): 218-229.

[83] Varma, M. V. S.; Sateesh, K.; Panchagnula, R. Functional role of P-glycoprotein in limiting intestinal absorption of drugs: Contribution of passive permeability to P-glycoprotein mediated efflux transport. Mol. Pharm., 2005, 2(1), 12-21.

[84] Kakumoto, M.; Takara, K.; Sakaeda, T.; Tanigawara, Y.; Kita, T.;Okumura, K. MDR1-mediated interaction of digoxin with antiarrhythmic or antianginal drugs. Biol. Pharm. Bull., 2002 25(12) 1604-1607.

[85] Broccatelli, F.; Larregieu, C. A.; Cruciani, G.; Oprea, T. I.; Benet, L. Z. Improving the prediction of the brain disposition for orally administered drugs using BDDCS. Adv. Drug Deliv. Rev., 2012, 64(1), 95-109. 